Proceedings of the Edinburgh Mathematical Society (2004) 47, 271-288 (C)

DOI:10.1017/S0013091502001050 Printed in the United Kingdom

\title{
ON BRANCHED COVERINGS OF LENS SPACES
}

\author{
ELENA BARBIERI AND FULVIA SPAGGIARI \\ Dipartimento di Matematica, Università di Modena e Reggio Emilia, \\ 41100 Modena, Italy (barbieri.elena@unimo.it; spaggiari.fulvia@unimo.it)
}

(Received 19 November 2002)

\begin{abstract}
We construct some series of polyhedral schemata which represent orientable closed connected 3 -manifolds. We show that these manifolds have spines corresponding to certain balanced presentations of their fundamental groups. Then we study some covering properties of such manifolds and prove that many of them are cyclic branched coverings of lens spaces. Our theorems contain a number of published results from various sources as particular cases.
\end{abstract}

Keywords: 3-manifold; group presentation; railroad system (RR-system); spine; polyhedral representation; branched covering

2000 Mathematics subject classification: Primary 57M12; 57M50; 57M60

\section{Introduction}

The goal of this paper is to study the topological and geometrical properties of certain classes of group presentations depending on a finite number of integers. This kind of work can be found in several papers (see, for example, $[\mathbf{1}-\mathbf{6}, \mathbf{8}-\mathbf{1 0}, \mathbf{1 2}-\mathbf{1 7}, \mathbf{1 9}, \mathbf{2 1}, \mathbf{2 7}, \mathbf{2 9}]$ ), where there are many connections between the theory of cyclically presented groups (see $\S 3$ for the definition) and the topology of cyclic branched coverings of knots. Given a knot $K$ in the 3 -sphere $\mathbb{S}^{3}$, we say that a closed connected 3-manifold $M$ is an $n$-fold cyclic branched covering of $K$ if $M$ is the $n$-fold cyclic covering of $\mathbb{S}^{3}$ branched over $K$ (see, for example, [26]). This implies that $M$ is the $n$-fold cyclic covering of the orbifold $\mathcal{O}(K, n)$ having $\mathbb{S}^{3}$ and $K$ as underlying space and singular set, respectively. In this case, the fundamental group $\pi_{1}(M)$ has a cyclic automorphism of order $n$, and the split-extension group of $\pi_{1}(M)$ is the group of the orbifold $\mathcal{O}(K, n)$. For example, if $K$ is the trefoil knot, then the fundamental group of the $n$-fold cyclic branched covering of $K$ is isomorphic to the Sieradski group $S(n)$ generated by $x_{1}, \ldots, x_{n}$ with cyclic relations $x_{i} x_{i+2}=x_{i+1}$, where the indices are taken $\bmod n$ (see [3]). If $K$ is the figure-eight knot, it was shown in [8] and [9] that the fundamental group of the $n$-fold cyclic branched covering of $K$ is isomorphic to the Fibonacci group $F(2,2 n)$ generated by $a_{1}, \ldots, a_{2 n}$ with cyclic relations $a_{i} a_{i+1}=a_{i+2}$ (indices $\bmod 2 n$ ). Moreover, a combinatorial description for such manifolds by Dehn surgeries on the components of certain oriented links was given in [2] (for surgery on links see also [20]). Sieradski and Fibonacci manifolds admit nice combinatorial representations as quotients of polyhedral 3-balls by pairwise identifications 
of oppositely oriented boundary faces. Some natural generalizations of these polyhedral schemata were given, for example, in $[\mathbf{1}, \mathbf{5}, \mathbf{1 2}, \mathbf{1 5}]$. In the present paper we will consider more general tessellations of the boundary of a 3-ball, including those obtained in the quoted papers as particular cases. These tessellations define a family of closed orientable 3-manifolds $M_{n}\left(p_{i}, q_{i}, k_{i}\right), i=1, \ldots, n$, having spines encoded by certain balanced group presentations. Our goal is to study some topological and geometrical properties of the constructed manifolds and groups.

For any $n \geqslant 1, k_{i} \in \mathbb{Z}$, and coprime integers $p_{i}, \ell_{i}\left(p_{i} \geqslant 1\right), i=1, \ldots, n$, we consider a family of finitely generated groups $G_{n}\left(p_{i}, \ell_{i}, k_{i}\right)$, which are defined by the following balanced presentations:

$$
\begin{aligned}
P_{n}\left(p_{i}, \ell_{i}, k_{i}\right)=\left\langle x_{1}, \ldots, x_{n}, y_{1}, \ldots, y_{n}: x_{i}^{-1} x_{i+1} y_{i}^{\ell_{i}} y_{i-1}^{-\ell_{i-1}+p_{i-1}}=1,\right. & \\
& \left.\left(y_{i}^{\ell_{i}} y_{i+1}^{-\ell_{i+1}}\right)^{k_{i}-1} y_{i}^{\ell_{i}} x_{i+2}=1(\operatorname{indices} \bmod n)\right\rangle .
\end{aligned}
$$

If $p_{i}=\ell_{i}=1$, the first relations give $y_{i}=x_{i+1}^{-1} x_{i}$ and substituting in the second relations yields

$$
P_{n}^{\prime}\left(1,1, k_{i}\right)=\left\langle x_{1}, \ldots, x_{n}:\left(x_{i+1}^{-1} x_{i} x_{i+1}^{-1} x_{i+2}\right)^{k_{i}-1} x_{i+1}^{-1} x_{i} x_{i+2}=1\right\rangle .
$$

These presentations were considered in [1] and [15] for $k_{i}=2$, and in [5] and [12] for $k_{i}=$ $k \geqslant 2$. It was shown that they correspond to spines of the $n$-fold cyclic branched coverings of the 2 -bridge knot $(2 k+1)_{2}$, which is the closure of the rational $(4 k-1) / 2$-tangle.

On the other hand, the second relations of $P_{n}\left(p_{i}, \ell_{i}, k_{i}\right)$ give

$$
x_{i+2}=y_{i}^{-\ell_{i}}\left(y_{i+1}^{\ell_{i+1}} y_{i}^{-\ell_{i}}\right)^{k_{i}-1}
$$

and substituting in the first relations yields new balanced presentations for the groups $G_{n}\left(p_{i}, \ell_{i}, k_{i}\right)$ :

$$
P_{n}^{\prime \prime}\left(p_{i}, \ell_{i}, k_{i}\right)=\left\langle y_{1}, \ldots, y_{n}:\left(y_{i}^{\ell_{i}} y_{i+1}^{-\ell_{i+1}}\right)^{k_{i}}\left(y_{i+2}^{\ell_{i+2}} y_{i+1}^{-\ell_{i+1}}\right)^{k_{i+1}} y_{i+1}^{p_{i+1}}=1\right\rangle
$$

(from now on the phrase 'indices $\bmod n$ ' will be understood). These presentations were considered in $[\mathbf{5}, \mathbf{1 3}, \mathbf{2 1}]$ for $p_{i}=p, \ell_{i}=q$ and $k_{i}=k$, and in $[\mathbf{1 2}]$ for $p_{i}=\ell_{i}=1$ and $k_{i}=k \geqslant 2$. It was shown that they define the fundamental groups of an interesting class of closed orientable 3-manifolds, called (periodic) Takahashi manifolds, first introduced in $[\mathbf{3 0}]$, and subsequently studied by several authors (see $[\mathbf{1 3}, \mathbf{2 1}, \mathbf{2 7}])$. Examples of Takahashi manifolds are given by Fibonacci manifolds $[\mathbf{2}, \mathbf{8}, \mathbf{9}]$, generalized Fibonacci manifolds $[\mathbf{1 6}, \mathbf{1 7}]$, fractional Fibonacci manifolds [14], and Sieradski manifolds $[\mathbf{3}, \mathbf{4}]$. As pointed out in [12], many Takahashi manifolds are also examples of maximally symmetric 3-manifolds in the sense of [32].

In $\S 2$ we briefly recall the representation theory of closed orientable 3 -manifolds by means of RR-systems, and use it to show that the group presentations defined above are geometric. In $\S 3$ we construct geometrically a family of closed orientable 3-manifolds by side pairings of oppositely oriented boundary faces of certain polyhedral 3-balls, and obtain spines corresponding to balanced presentations of their fundamental groups. Finally, $\S 4$ is devoted to a study of some covering properties of such manifolds, and split extensions of their fundamental groups. 


\section{Geometric presentations}

In this section we show that the group presentations $P_{n}^{\prime \prime}\left(p_{i}, \ell_{i}, k_{i}\right)$ are geometric, that is, they correspond to spines of closed connected orientable 3 -manifolds. To prove this, we use the combinatorial representations of closed orientable 3-manifolds by RR-systems (railroad systems), due to Osborne and Stevens (see $[\mathbf{2 2 - 2 5}, \mathbf{2 9}]$ ), and subsequently completed by Hog-Angeloni in [11]. We briefly sketch the theory from a qualitative point of view by making use of an example arising from our geometric constructions (more details and precise definitions can be found in the quoted papers). So, let $P$ be a finite balanced group presentation with $n$ generators $x_{i}$ and $n$ relators $r_{i}$. Let $K_{P}$ denote the canonical 2-complex (with one vertex) associated with $P$. It is well known that any closed connected orientable 3-manifold admits a spine of type $K_{P}$, but not every 2-complex $K_{P}$ is a spine of some 3-manifold. There are many criteria in the literature for $P$ to be a geometric presentation. The first one is based on the representation of 3-manifolds by Heegaard diagrams. Let $M$ be a closed connected orientable 3-manifold, and let $\left(V_{1}, V_{2}\right)$ denote a Heegaard splitting of $M$ of genus $n$. A properly embedded disc $D$ in the handlebody $V_{2}$ is called a meridian disc of $V_{2}$ if cutting $V_{2}$ along $D$ yields a handlebody of genus $n-1$. A collection of $n$ mutually disjoint meridian discs $\left\{D_{i}\right\}$ in $V_{2}$ is called a complete system of meridian discs of $V_{2}$ if cutting $V_{2}$ along $\bigcup_{i} D_{i}$ gives a 3-ball. Let $\alpha_{i}$ denote the 1-sphere $\partial D_{i}$ which lies in the closed orientable surface $\partial V_{1}=\partial V_{2}$ of genus $n$. The system $\left(V_{1} ; \alpha_{1}, \ldots, \alpha_{n}\right)$ is said to be a Heegaard diagram of $M$ associated with the splitting $\left(V_{1}, V_{2}\right)$.

The following criterion is well known (see, for example, [11]).

Theorem 2.1. Let $P$ be a finite balanced group presentation with $n$ generators $x_{i}$ and $n$ relators $r_{i}$. The canonical 2-complex $K_{P}$ is a spine of a closed orientable 3-manifold $M$ if and only if it is possible to draw 2-sided simple closed curves $\alpha_{1}, \ldots, \alpha_{n}$ on the boundary of an orientable handlebody $V$ with $n$ handles (one handle for each generator $x_{i}$ ) such that $\alpha_{i}$ reads the relator $r_{i}$.

Let us consider, for example, the group presentation $P=P_{2}^{\prime \prime}\left(p_{i}, \ell_{i}, k_{i}\right)$, where $\left(p_{1}, \ell_{1}\right)=$ $(2,-1),\left(p_{2}, \ell_{2}\right)=(3,1)$ and $k_{1}=k_{2}=1$. Then we have

$$
P=\left\langle x, y: y^{3}=(x y)^{2}=x^{-2}\right\rangle .
$$

This presentation is geometric since it arises from the Heegaard diagram drawn in Figure 1 . Then $K_{P}$ corresponds to a spine of a closed orientable 3-manifold. The curves $\alpha_{1}$ and $\alpha_{2}$ on the boundary of a genus 2 orientable handlebody read the relators $(x y)^{2} x^{2}$ and $y^{3} x^{2}$, respectively.

Cutting the surface $F=\partial V$ along a system of meridian discs determines a 2-sphere with $2 n$ holes. These holes correspond to pairs of attaching discs, $D_{i}$ and $\bar{D}_{i}$ say, arising from the cutting of the handles of $V$. Any curve $\alpha_{i}$ specified above splits into a finite collection of pairwise-disjoint simple arcs $\left\{\alpha_{i j}\right\}$ running on the punctured 2-sphere. We can always assume that these arcs are transversal to the boundaries of the holes. For example, the Heegaard diagram of Figure 1 can be represented on the punctured 2sphere, as shown in Figure 2. 


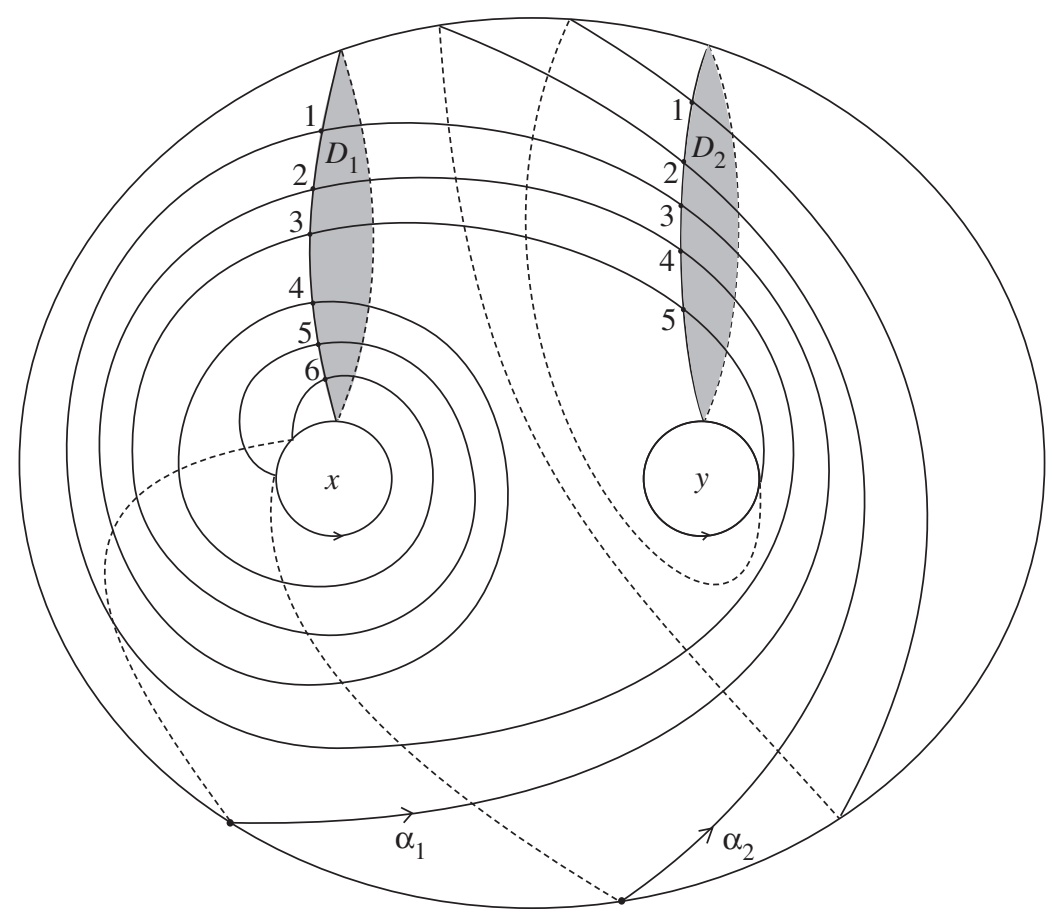

Figure 1. A Heegaard diagram of $M=(O 0 O:-1(2,-1)(3,1)(2,1))$ inducing the presentation $\left\langle x, y:(x y)^{2}=x^{-2}=y^{3}\right\rangle$.

Of course, we can restate Theorem 2.1 in the following equivalent form that uses graphical representations on punctured spheres.

Theorem 2.2. With the above notation, $K_{P}$ is a spine of a closed orientable 3-manifold $M$ if and only if it is possible to draw $n$ collections $\boldsymbol{\alpha}_{i}=\left\{\alpha_{i j}\right\}$ of pairwise-disjoint simple arcs on a 2 -sphere with $2 n$ holes such that $\boldsymbol{\alpha}_{i}$ reads the relator $r_{i}$.

It turns out that each 'hole' $D_{i}$ on the punctured 2-sphere can be regarded as a hexagon labelled by the generator $x_{i}$ of $P$. The edges of this hexagon are labelled by integers $p_{i}$, $\ell_{i}$ and $p_{i}+\ell_{i}$, where $p_{i}$ and $\ell_{i}$ are coprime. The arcs of the collections $\boldsymbol{\alpha}_{i}=\left\{\alpha_{i j}\right\}$ connect the hexagons. Their endpoints avoid the corners of the hexagons. We run from an arc $\alpha_{i j}$ to the next one $\alpha_{i j+1}$ by proceeding along a line segment which lies in the interior of a hexagon and is orthogonal to two of its edges. To obtain the relator $r_{i}$ corresponding to the collection $\boldsymbol{\alpha}_{i}=\left\{\alpha_{i j}\right\}$, it suffices to read the labels of the edges of the hexagons encountered running along the arcs of the collection. This construction, which gives rise to an RR-system (railroad system), is shown in Figure 3 for our initial example.

The following is a fundamental result in the theory of RR-systems (see the quoted papers).

Theorem 2.3. The complex $K_{P}$ is a spine of a closed connected orientable 3-manifold if and only if it is possible to draw collections $\boldsymbol{\alpha}_{i}$ of pairwise-disjoint simple arcs on a 


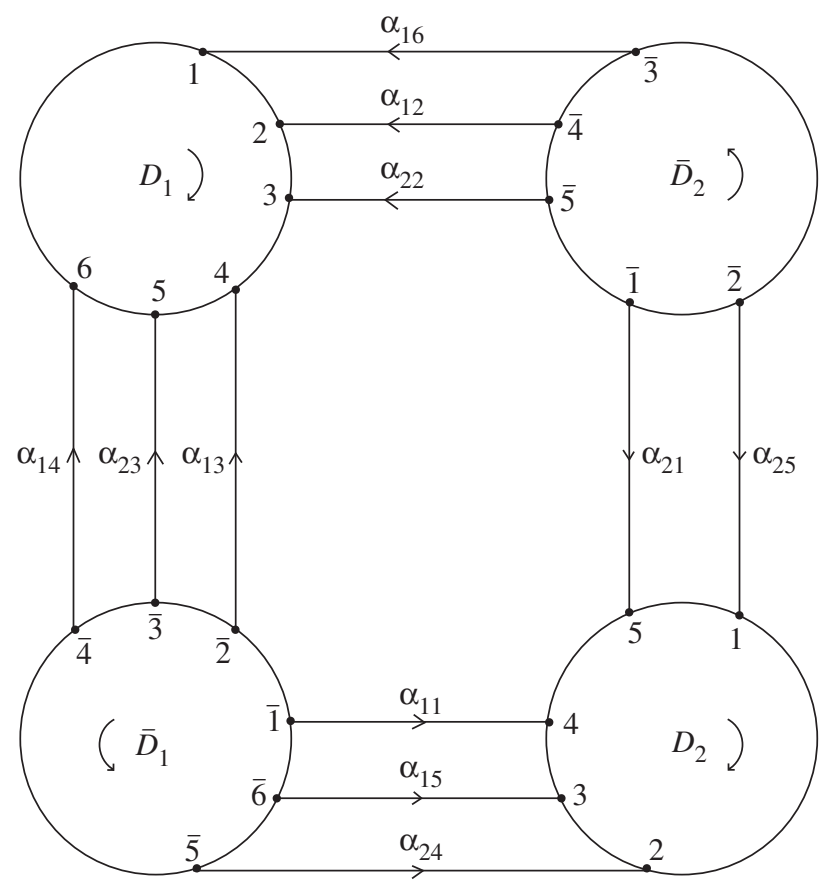

Figure 2. The Heegaard diagram of $M=(O 0 O:-1(2,-1)(3,1)(2,1))$ drawn on the punctured 2-sphere.

2-sphere with $n$ hexagons labelled by integers $p_{i}, \ell_{i}$ and $p_{i}+\ell_{i}$, where $p_{i}$ and $\ell_{i}$ are coprime (briefly, an $R R$-system), such that $\boldsymbol{\alpha}_{i}$ reads the relator $r_{i}$.

As an application, we state a nice result that follows immediately from Theorem 3.1 of [22] (cf. also the subsequent remark on p. 485).

Proposition 2.4. Let $M$ be a closed connected orientable 3-manifold having a spine which corresponds to the geometric presentation

$$
\left\langle x, y: x^{p} y^{n}=1,\left(x^{m} y^{q}\right)^{k} x^{m} y^{n+q}=1\right\rangle,
$$

where $|p|,|n|>1$, and $p, m$, respectively, $n, q$, are coprime integers. Then $M$ is homeomorphic to the Seifert fibred manifold defined by the invariants

$$
(O 0 O:-1(p, m)(n,-q)(k+1, k)) .
$$

Now let us consider the RR-system depicted in Figure 4. Then it induces a balanced presentation with three generators $y_{1}, y_{2}$ and $y_{3}$ and three relations (indices mod 3 )

$$
\left(y_{i}^{\ell_{i}} y_{i+1}^{-\ell_{i+1}}\right)^{k_{i}}\left(y_{i+2}^{\ell_{i+2}} y_{i+1}^{-\ell_{i+1}}\right)^{k_{i+1}} y_{i+1}^{p_{i+1}}=1,
$$

where $k_{1}=1, k_{2}=2$ and $k_{3}=3$ (one can directly draw pictures of RR-systems for the general case by simple iterations). If $p_{i}$ and $\ell_{i}$ are coprime, Theorem 2.3 implies that these presentations are geometric, and so they correspond to spines of closed orientable 3-manifolds. More precisely, we have the following theorem. 


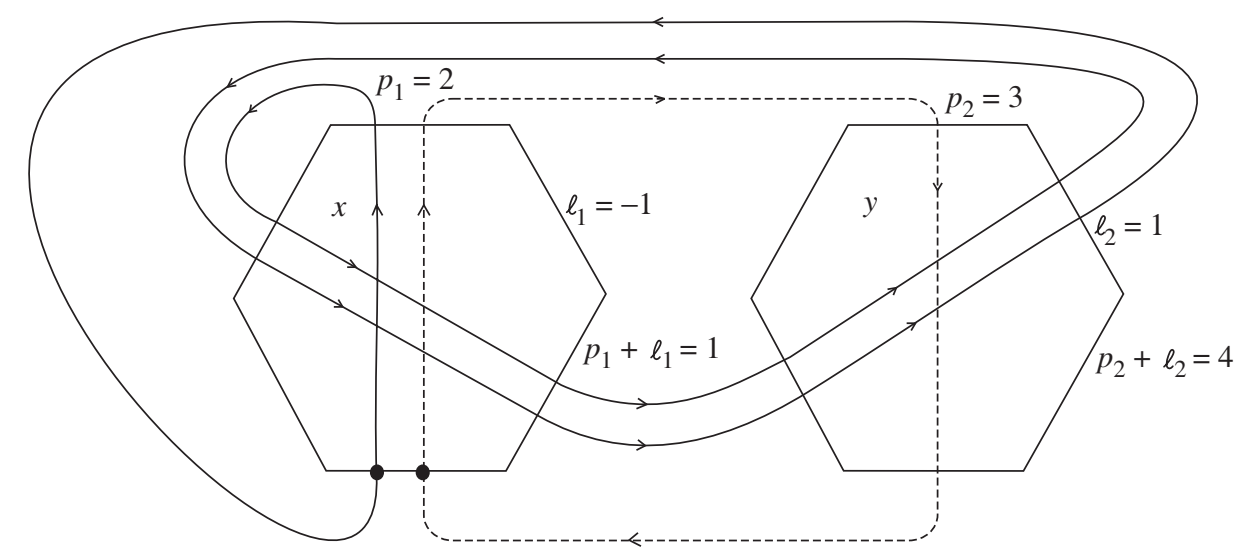

Figure 3. An RR-system inducing the group presentation $\left\langle x, y:(x y)^{2}=x^{-2}=y^{3}\right\rangle$.

Theorem 2.5. For all coprime integers $p_{i}$ and $\ell_{i}\left(p_{i} \geqslant 1\right)$ and $k_{i} \in \mathbb{Z}$, the balanced group presentations $P_{n}^{\prime \prime}\left(p_{i}, \ell_{i}, k_{i}\right)$ arise from $R R$-systems, that is, they are geometric. Then the canonical 2-complexes corresponding to them are spines of closed connected orientable 3-manifolds $M_{n}\left(p_{i}, \ell_{i}, k_{i}\right)$.

We will see in $\S 4$ that the manifold $M_{n}\left(p_{i}, \ell_{i}, k_{i}\right)$ for $n=1$ is homeomorphic to the lens space $L(p, \ell)$ (including the 3 -sphere for $p=1$ ), where $p_{i}=p$ and $\ell_{i}=\ell$.

For $n=2$, the group presentation $P_{n}^{\prime \prime}\left(p_{i}, \ell_{i}, k_{i}\right)$ becomes

$$
\begin{aligned}
P_{2}^{\prime \prime}=P_{2}^{\prime \prime}\left(p_{i}, \ell_{i}, k_{i}\right)=\left\langle y_{1}, y_{2}:\left(y_{1}^{\ell_{1}} y_{2}^{-\ell_{2}}\right)^{k_{1}}\left(y_{1}^{\ell_{1}} y_{2}^{-\ell_{2}}\right)^{k_{2}} y_{2}^{p_{2}}=1,\right. \\
\\
\left.\left(y_{2}^{\ell_{2}} y_{1}^{-\ell_{1}}\right)^{k_{2}}\left(y_{2}^{\ell_{2}} y_{1}^{-\ell_{1}}\right)^{k_{1}} y_{1}^{p_{1}}=1\right\rangle ;
\end{aligned}
$$

hence

$$
\begin{aligned}
P_{2}^{\prime \prime} & =\left\langle y_{1}, y_{2}: y_{1}^{p_{1}}=\left(y_{1}^{\ell_{1}} y_{2}^{-\ell_{2}}\right)^{k_{1}+k_{2}}=y_{2}^{-p_{2}}\right\rangle \\
& \cong\left\langle y_{1}, y_{2}: y_{1}^{p_{1}} y_{2}^{p_{2}}=1,\left(y_{1}^{\ell_{1}} y_{2}^{-\ell_{2}}\right)^{k_{1}+k_{2}-1} y_{1}^{\ell_{1}} y_{2}^{p_{2}-\ell_{2}}=1\right\rangle .
\end{aligned}
$$

Since this presentation is geometric, we apply Proposition 2.4 to get the following result.

Proposition 2.6. For any coprime integers $p_{i}$ and $\ell_{i}\left(p_{i}>1\right)$ and $k_{i} \in \mathbb{Z}$, the manifold $M_{2}\left(p_{i}, \ell_{i}, k_{i}\right)$ is homeomorphic to the Seifert fibred space defined by the invariants

$$
\left(O 0 O:-1\left(p_{1}, \ell_{1}\right)\left(p_{2}, \ell_{2}\right)\left(k_{1}+k_{2}, k_{1}+k_{2}-1\right)\right) .
$$

In particular, our example is the spherical Seifert manifold defined by the invariants $(O 0 O:-1(2,-1)(3,1)(2,1))$. By $[\mathbf{2 8}]$ we recall that the geometry of a Seifert bundle can be determined from the invariants $\chi$ and $e$, where $\chi$ is the Euler characteristic of the base orbifold and $e$ is the Euler number of the Seifert bundle. For the manifolds $M_{2}\left(p_{i}, \ell_{i}, k_{i}\right)$ we have

$$
e=\frac{1}{k_{1}+k_{2}}-\frac{\ell_{1}}{p_{1}}-\frac{\ell_{2}}{p_{2}}, \quad \chi=-1+\frac{1}{k_{1}+k_{2}}+\frac{1}{p_{1}}+\frac{1}{p_{2}} .
$$

As a consequence, the geometric structures of such manifolds are completely determined as follows. 


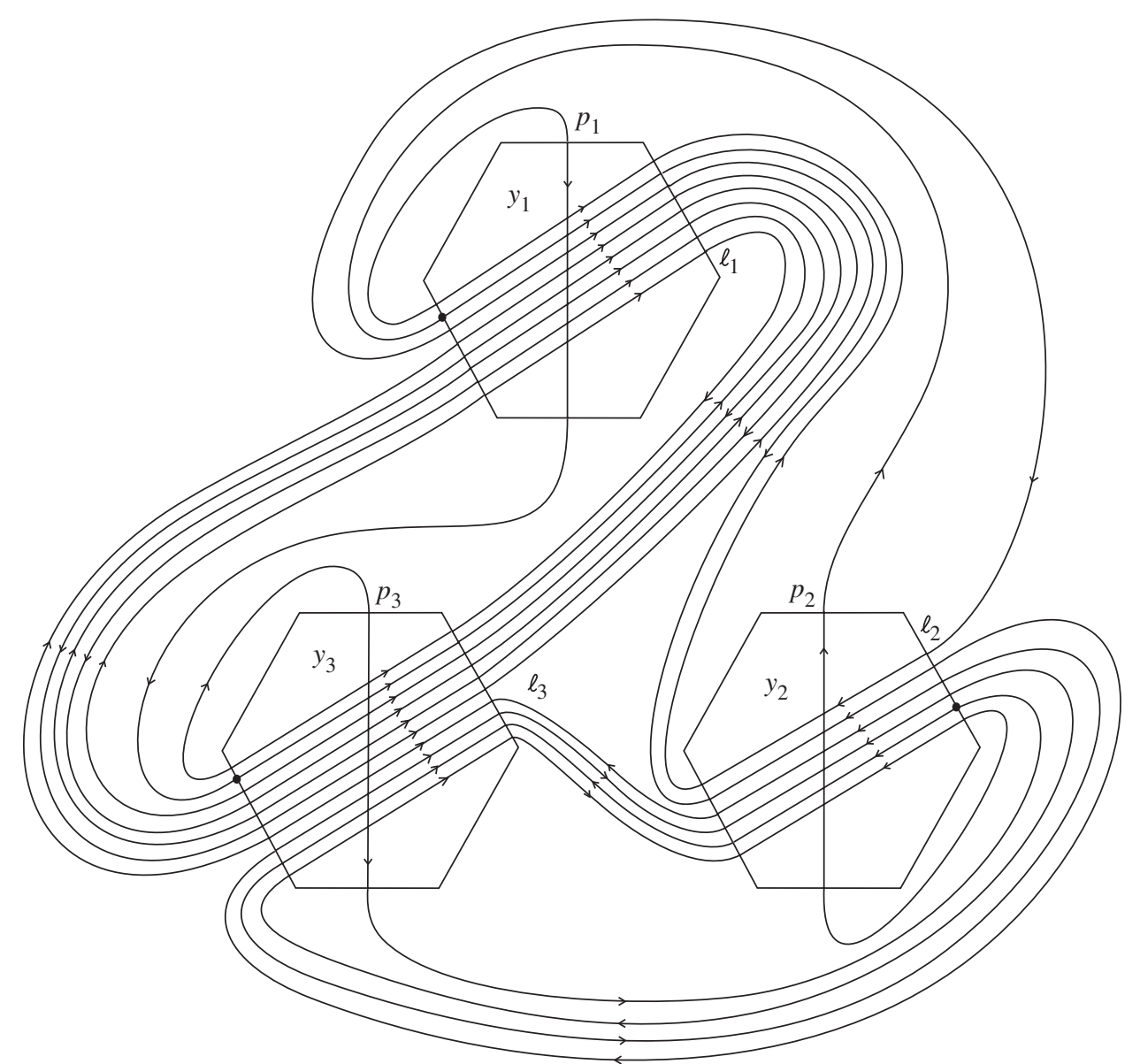

Figure 4. An RR-system inducing the presentations $P_{n}^{\prime \prime}\left(p_{i}, \ell_{i}, k_{i}\right)$ (case $n=3, k_{1}=1, k_{2}=2, k_{3}=3$ ).

Corollary 2.7. If

$$
p_{1} p_{2}=\left(k_{1}+k_{2}\right)\left(p_{1} \ell_{2}+p_{2} \ell_{1}\right)
$$

and $\chi<0$ (respectively, $\chi=0, \chi>0$ ), then $M_{2}\left(p_{i}, \ell_{i}, k_{i}\right)$ is an $\mathbb{H}^{2} \times \mathbb{R}$-manifold (respectively, a Euclidean manifold, a $\mathbb{S}^{2} \times \mathbb{R}$-manifold). If

$$
p_{1} p_{2} \neq\left(k_{1}+k_{2}\right)\left(p_{1} \ell_{2}+p_{2} \ell_{1}\right)
$$

and $\chi<0$ (respectively, $\chi=0, \chi>0)$, then $M_{2}\left(p_{i}, \ell_{i}, k_{i}\right)$ is a $\widetilde{\mathrm{SL}_{2}(\mathbb{R})}$-manifold (respectively, a nil-manifold, a spherical manifold).

\section{Polyhedral constructions}

In this section we construct a nice polyhedron $\mathbb{P}_{n}\left(p_{i}, \ell_{i}, k_{i}\right)$ (which is homeomorphic to a 3 -ball) representing the closed orientable manifold $M_{n}\left(p_{i}, \ell_{i}, k_{i}\right)$. It turns out that 
the fundamental group of this manifold is isomorphic to the group $G_{n}\left(p_{i}, \ell_{i}, k_{i}\right)$ defined above. Moreover, the canonical 2-complex associated with the balanced presentation $P_{n}\left(p_{i}, \ell_{i}, k_{i}\right)$ is a spine of the manifold $M_{n}\left(p_{i}, \ell_{i}, k_{i}\right)$. So this presentation is geometric, and arises from a Heegaard diagram of the manifold which can be immediately obtained from its polyhedral representation.

Theorem 3.1. For $n \geqslant 1, k_{i} \in \mathbb{Z}, k_{i} \geqslant 2$, and coprime integers $p_{i}, \ell_{i}, 1 \leqslant p_{i}<\ell_{i}$, the balanced presentation $P_{n}\left(p_{i}, \ell_{i}, k_{i}\right)$ of the group $G_{n}\left(p_{i}, \ell_{i}, k_{i}\right)$ corresponds to a spine of a closed connected orientable 3 -manifold $M_{n}\left(p_{i}, \ell_{i}, k_{i}\right)$.

Proof. Let us consider a triangulated 3 -ball $\mathbb{P}_{n}\left(p_{i}, \ell_{i}, k_{i}\right)$ whose boundary consists of $n$ polygons $T_{i}^{\prime}$ of $\ell_{i}+\ell_{i-1}-p_{i-1}+2$ edges in the northern hemisphere, $n$ polygons $Q_{i}^{\prime}$ of $k_{i} \ell_{i}+\left(k_{i}-1\right) \ell_{i+1}+1$ edges in the southern hemisphere, and polygons $T_{i}$ and $Q_{i}$ having the same number of edges of $T_{i}^{\prime}$ and $Q_{i}^{\prime}$, respectively, in the equatorial zone $(i=1, \ldots, n$; indices $\bmod n)$. For example, the polyhedron $\mathbb{P}_{3}\left(p_{i}, \ell_{i}, k_{i}\right)$, where $\operatorname{gcd}\left(p_{i}, \ell_{i}\right)=1, \ell_{i}>p_{i}$ $(i=1,2,3), k_{1}=2, k_{2}=3$ and $k_{3}=4$ is depicted in Figure 5 . Here we have used a simplified notation, that is, an 'edge' labelled, for example, by a symbol $y_{i}^{\ell_{i}-p_{i}}$ represents a sequence of $\ell_{i}-p_{i}$ equally oriented edge segments labelled by $y_{i}$.

If the parameters satisfy the conditions listed above, the oriented edges fall into $2 n$ classes labelled $x_{1}, \ldots, x_{n}$ and $y_{1}, \ldots, y_{n}$.

For each $i=1, \ldots, n$, the boundary cycle of the polygons $T_{i}$ and $T_{i}^{\prime}$ is given by the word

$$
x_{i}^{-1} x_{i+1} y_{i}^{\ell_{i}} y_{i-1}^{-\ell_{i-1}+p_{i-1}},
$$

where the indices are taken $\bmod n$. For each $i=1, \ldots, n$, the boundary cycle of the polygons $Q_{i}$ and $Q_{i}^{\prime}$ is defined by the word

$$
\left(y_{i}^{\ell_{i}} y_{i+1}^{-\ell_{i+1}}\right)^{k_{i}-1} y_{i}^{\ell_{i}} x_{i+2},
$$

where the indices are taken $\bmod n$. Now we consider the pairwise identifications of the boundary faces of the polyhedron $\mathbb{P}_{n}\left(p_{i}, \ell_{i}, k_{i}\right)$. For each $i=1, \ldots, n$, let $t_{i}$ identify the polygons $T_{i}$ and $T_{i}^{\prime}$, and let $q_{i}$ identify the polygons $Q_{i}$ and $Q_{i}^{\prime}$ so that the corresponding oriented edges of the polygons carrying the same label are identified together. As a result of this side pairing, we get a cellular complex which triangulates an orientable pseudomanifold $M_{n}\left(p_{i}, \ell_{i}, k_{i}\right)$. This quotient complex has one vertex, $2 n$ edges, $2 n$ 2-cells, and one 3-cell. So it is a closed connected orientable 3-manifold, as its Euler characteristic vanishes. The fundamental group of this manifold is isomorphic to the group $G_{n}\left(p_{i}, \ell_{i}, k_{i}\right)$. Furthermore, the balanced presentation $P_{n}\left(p_{i}, \ell_{i}, k_{i}\right)$ corresponds to a spine of the constructed manifold.

Let us consider now the particular case $p_{i}=1, \ell_{i}=\ell \geqslant 1$ and $k_{i}=k \geqslant 2$, and denote the polyhedron $\mathbb{P}_{n}\left(p_{i}, \ell_{i}, k_{i}\right)$ simply by $\mathbb{P}_{n}(k, \ell)$. The boundary of this polyhedron consists of $n(2 \ell+1)$-gons

$$
T_{i}^{\prime}=N H_{i} \cdots A_{i} A_{i+1} \cdots H_{i+1}
$$




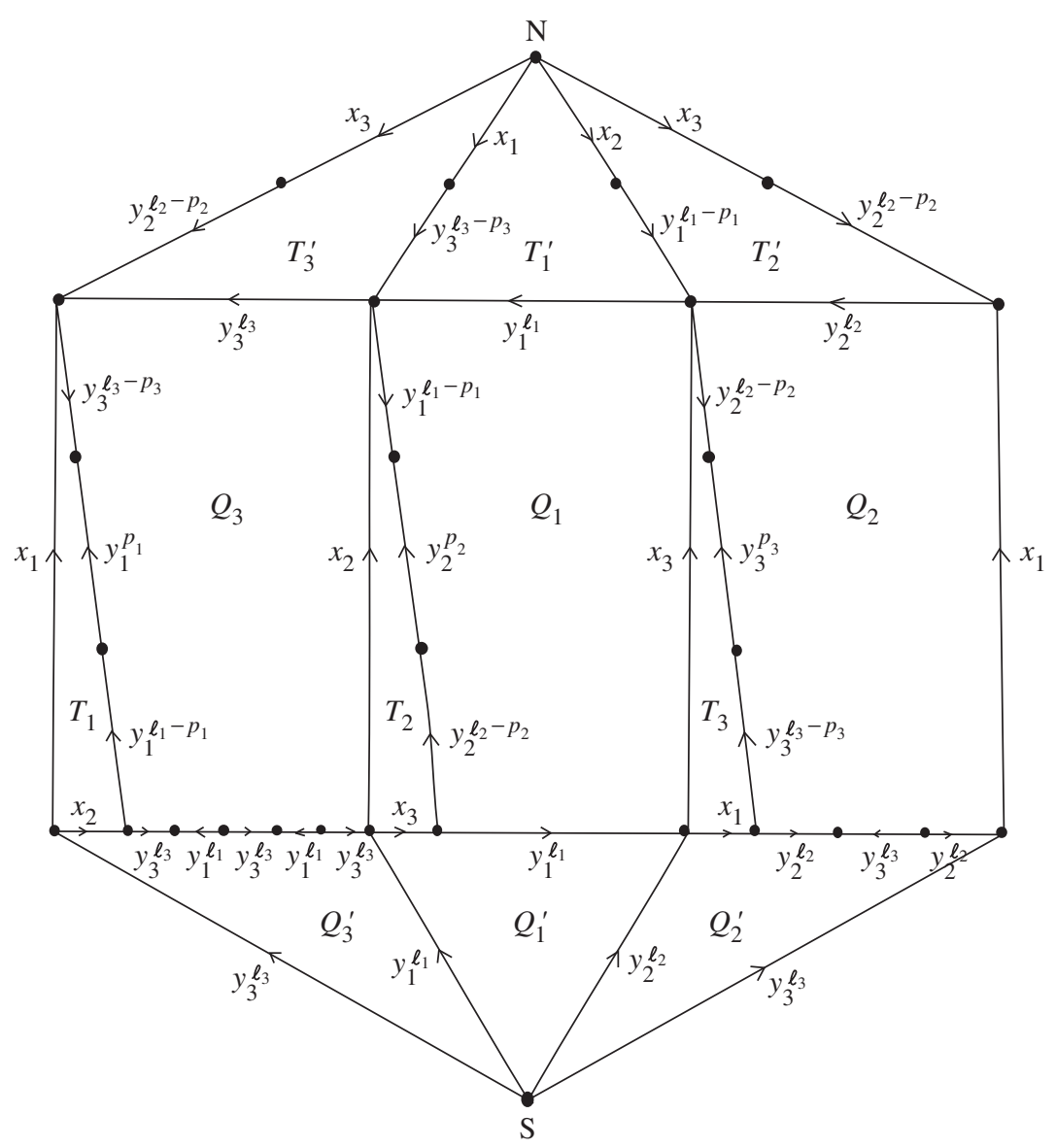

Figure 5. The polyhedron $\mathbb{P}_{3}\left(p_{i}, \ell_{i}, k_{i}\right), \ell_{i}>p_{i}, \operatorname{gcd}\left(p_{i}, \ell_{i}\right)=1, k_{1}=2, k_{2}=3$ and $k_{3}=4$.

in the northern hemisphere, $n[\ell(2 k-1)+1]$-gons

$$
Q_{i}^{\prime}=S \cdots K_{i} B_{i} C_{i} D_{i} \cdots B_{i+1}
$$

in the southern hemisphere, $n(2 \ell+1)$-gons

$$
T_{i}=A_{i-1} B_{i-1} C_{i-1} L_{i-1} \cdots M_{i-1},
$$

and $n[\ell(2 k-1)+1]$-gons

$$
Q_{i}=A_{i} A_{i+1} B_{i+1} \cdots C_{i} L_{i} \cdots M_{i}
$$

in the equatorial zone, where $i=1, \ldots, n$, and all indices are taken $\bmod n$. Thus $\mathbb{P}_{n}(k, \ell)$ has $4 n$ faces, $(2 \ell+1) n+n[\ell(2 k-1)+1]$ edges, and $\ell n(2 k+1)+2-2 n$ vertices. For example, the polyhedron $\mathbb{P}_{3}(3,2)$ is depicted in Figure 6 .

If $\ell=1$ and $k=2$, we obtain exactly the polyhedra considered in [1] and [15], whose side pairing quotients triangulate the cyclic branched coverings of the knot $5_{2}$. If $\ell=1$ 


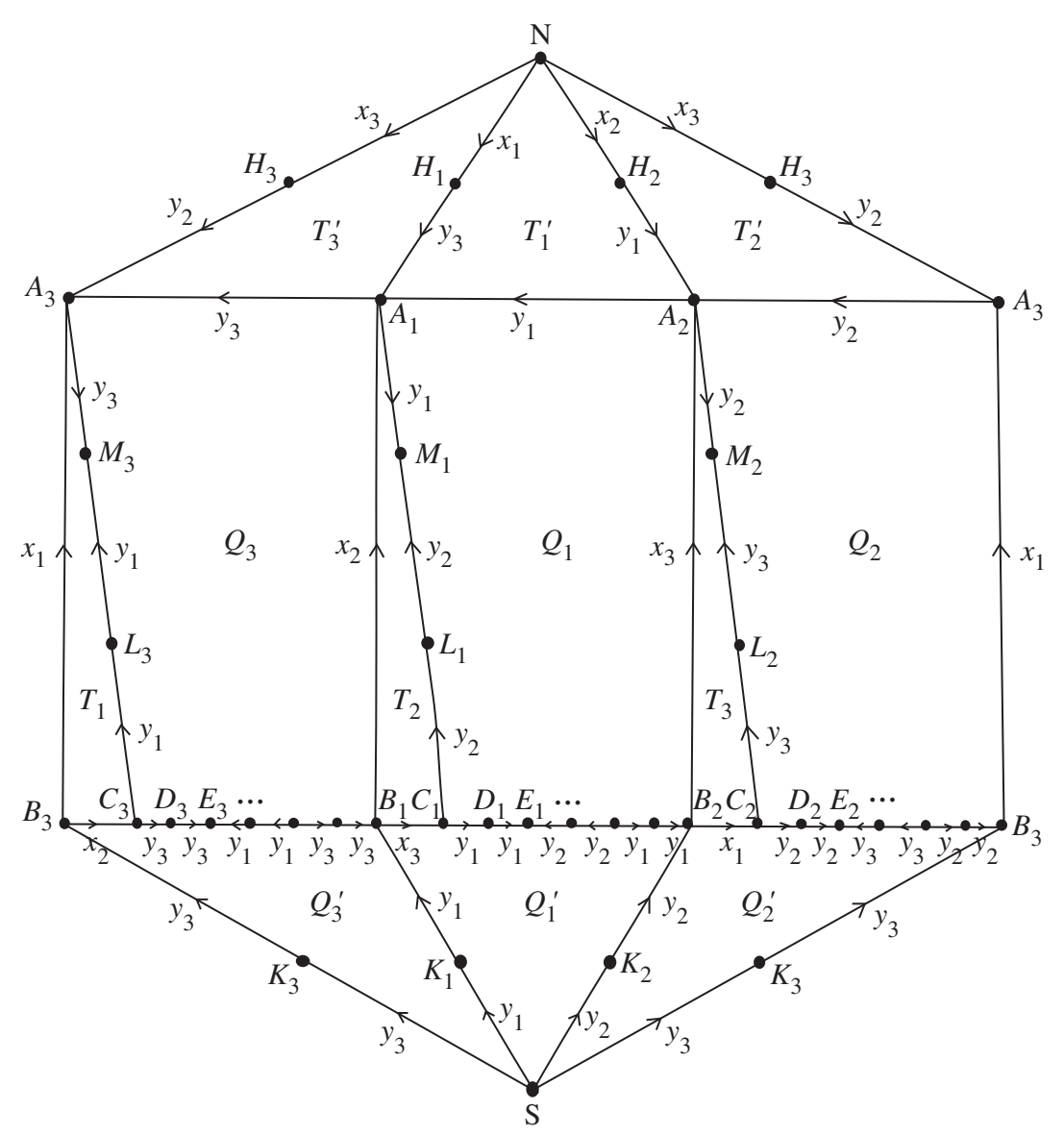

Figure 6 . The polyhedron $\mathbb{P}_{3}(k, \ell)$, where $k=3$ and $\ell=2$.

and $k \geqslant 2$, then we have the polyhedra constructed in [5] and [12], which give polyhedral schemata for cyclic branched coverings of the knot $(2 k+1)_{2}$.

By side pairings, the oriented edges fall into $2 n$ classes labelled $x_{1}, \ldots, x_{n}$ (each one of them formed by exactly three edges), and $y_{1}, \ldots, y_{n}$ (each one of them consisting of $\ell(2 k+1)-1$ edges). For each $i=1, \ldots, n$, the boundary cycle of the $(2 \ell+1)$-gons $T_{i}$ and $T_{i}^{\prime}$ is given by the word $x_{i}^{-1} x_{i+1} y_{i}^{\ell} y_{i-1}^{-\ell+1}$, where the indices are taken $\bmod n$. For each $i=1, \ldots, n$, the boundary cycle of the $[\ell(2 k-1)+1]$-gons $Q_{i}$ and $Q_{i}^{\prime}$ is defined by the word $\left(y_{i}^{\ell} y_{i+1}^{-\ell}\right)^{k-1} y_{i}^{\ell} x_{i+2}$, where the indices are taken $\bmod n$. Now we consider the pairwise identifications of the boundary faces of the polyhedron $\mathbb{P}_{n}(k, \ell)$. For each $i=1, \ldots, n$, let $t_{i}$ identify the $(2 \ell+1)$-gons $T_{i}$ and $T_{i}^{\prime}$, and let $q_{i}$ identify the $[\ell(2 k-1)+1]$-gons $Q_{i}$ and $Q_{i}^{\prime}$ so that the corresponding oriented edges of the polygons carrying the same label are identified together. This produces cycles of equivalent edges as follows. For the label $x_{i}$, we have

$$
B_{i-1} A_{i-1} \stackrel{t_{i}}{\longrightarrow} N H_{i} \stackrel{t_{i-1}^{-1}}{\longrightarrow} B_{i-2} C_{i-2} \stackrel{q_{i-2}^{-1}}{\longrightarrow} B_{i-1} A_{i-1},
$$


whence

$$
t_{i} t_{i-1}^{-1} q_{i-2}^{-1}=1
$$

For the label $y_{i}$, we get

$$
\begin{aligned}
& A_{i+1} A_{i} \stackrel{q_{i}}{\longrightarrow} C_{i} D_{i} \stackrel{q_{i}}{\longrightarrow} \cdots \stackrel{q_{i}}{\longrightarrow} S K_{i} \stackrel{q_{i-1}^{-1}}{\longrightarrow} \cdots \\
& \stackrel{q_{i-1}^{-1}}{\longrightarrow} C_{i-1} L_{i-1} \stackrel{t_{i}}{\longrightarrow} H_{i+1} A_{i+1} \stackrel{t_{i+1}^{-1}}{\longrightarrow} A_{i} M_{i} \stackrel{q_{i}}{\longrightarrow} \cdots \\
& \stackrel{q_{i}}{\longrightarrow} K_{i} B_{i} \stackrel{q_{i-1}^{-1}}{\longrightarrow} \cdots \stackrel{q_{i-1}^{-1}}{\longrightarrow} L_{i-1} M_{i-1} \stackrel{t_{i}}{\longrightarrow} A_{i+1} A_{i},
\end{aligned}
$$

whence

$$
\left(q_{i}^{k} q_{i-1}^{-(k-1)} t_{i} t_{i+1}^{-1}\right)^{\ell-1} q_{i}^{k} q_{i-1}^{-(k-1)} t_{i}=1 .
$$

Let us denote the quotient manifold simply by $M_{n}(k, \ell)$. So we have proved the following corollary which extends a result of [15] (case $\ell=1$ and $k=2$ ).

Corollary 3.2. For any $n \geqslant 1, \ell \geqslant 1$ and $k \geqslant 2$, the fundamental group of the manifold $M_{n}(k, \ell)$ admits the balanced presentation (which is the dual of the presentation $P_{n}(k, \ell$ ) of the group $\left.G_{n}(k, \ell)\right)$

$$
P_{n}^{*}(k, \ell)=\left\langle t_{1}, \ldots, t_{n}, q_{1}, \ldots, q_{n}: t_{i} t_{i-1}^{-1} q_{i-2}^{-1}=1,\left(q_{i}^{k} q_{i-1}^{-(k-1)} t_{i} t_{i+1}^{-1}\right)^{\ell} t_{i+1}=1\right\rangle .
$$

The first relations give $q_{i}=t_{i+2} t_{i+1}^{-1}$ and substituting into the second relations yields

$$
\left(\left(t_{i+2} t_{i+1}^{-1}\right)^{k}\left(t_{i} t_{i+1}^{-1}\right)^{k-1} t_{i} t_{i+1}^{-1}\right)^{\ell} t_{i+1}=1
$$

hence we get a further consequence as follows.

Corollary 3.3. With the above notation, the group $G_{n}(k, \ell)$ admits the cyclic presentation

$$
\left\langle t_{1}, \ldots, t_{n}:\left(\left(t_{i+2} t_{i+1}^{-1}\right)^{k}\left(t_{i} t_{i+1}^{-1}\right)^{k}\right)^{\ell} t_{i+1}=1\right\rangle,
$$

which is isomorphic to the cyclic presentation

$$
P_{n}^{\prime \prime}(k, \ell)=\left\langle y_{1}, \ldots, y_{n}:\left(y_{i}^{\ell} y_{i+1}^{-\ell}\right)^{k}\left(y_{i+2}^{\ell} y_{i+1}^{-\ell}\right)^{k} y_{i+1}=1\right\rangle
$$

obtained in $\S 1$.

\section{Split extensions and branched coverings}

In this section we consider the manifolds $M_{n}\left(p_{i}, \ell_{i}, k_{i}\right)$, where $p_{i}=p \geqslant 1, \ell_{i}=\ell \geqslant 1$, $\operatorname{gcd}(p, \ell)=1$ and $k_{i}=k \geqslant 2$. We denote such manifolds briefly by $M_{n}(k, \ell, p)$ (and $M_{n}(k, \ell)$ as usual for $\left.p=1\right)$. We will prove that these manifolds are cyclic branched coverings of the lens space $L(p, \ell)$ (including the 3 -sphere for $p=1$ ). This gives explicit examples of $L(p, \ell)$-hyperelliptic manifolds in the sense of [18]. We also study the split-extension group of $G_{n}(k, \ell)$ by the cyclic automorphism corresponding to cyclic presentations of it. For this, we recall a few definitions on group presentations and 
refer to any standard textbook for more details. Let $F_{n}$ be the free group of rank $n$ generated by $x_{1}, \ldots, x_{n}$, and let $\theta$ be the automorphism of $F_{n}$ defined by setting $\theta\left(x_{i}\right)=x_{i+1}$, for $i=1, \ldots, n$, where the indices are taken $\bmod n$. For a reduced word $w$ in $F_{n}$, let $G_{n}(w)$ be the factor group $F_{n} / R$, where $R$ is the normal closure in $F_{n}$ of the set $\left\{w, \theta(w), \ldots, \theta^{n-1}(w)\right\}$. Then $G_{n}(w)$ has a cyclic presentation with generators $x_{1}, \ldots, x_{n}$, and relations $w=1, \theta(w)=1, \ldots, \theta^{n-1}(w)=1$. A group $G$ is said to have a cyclic presentation if $G$ is isomorphic to $G_{n}(w)$ for some $w$ and $n$. Of course, $\theta$ induces an automorphism of $G_{n}(w)$ which determines an action of the cyclic group $\mathbb{Z}_{n}=\left\langle\theta: \theta^{n}=1\right\rangle$ on $G_{n}(w)$. The split-extension group $H_{n}(v)$ of $G_{n}(w)$ by $\mathbb{Z}_{n}$ admits a presentation with generators $x$ and $\theta$, and relations $\theta^{n}=1$ and $v(\theta, x)=1$, where $v(\theta, x)=w\left(x, \theta^{-1} x \theta, \ldots, \theta^{-(n-1)} x \theta^{n-1}\right)$.

Let us consider the polyhedron $\mathbb{P}_{n}(k, \ell)$, and let $\rho$ be the symmetry of order $n$ of $\mathbb{P}_{n}(k, \ell)$ such that

$$
\rho: T_{i} \rightarrow T_{i+1}, T_{i}^{\prime} \rightarrow T_{i+1}^{\prime}, Q_{i} \rightarrow Q_{i+1}, Q_{i}^{\prime} \rightarrow Q_{i+1}^{\prime},
$$

where the indices are taken $\bmod n$. With respect to the presentation of $G_{n}(k, \ell)$ written in Corollary 3.2, the action of the automorphism $\rho$ is given by $t_{i} \rightarrow t_{i+1}$ and $q_{i} \rightarrow q_{i+1}$. Let $H_{n}(k, \ell)$ denote the split-extension group of $G_{n}(k, \ell)$ by the cyclic group $\mathbb{Z}_{n}$ generated by $\rho$.

Theorem 4.1. For $n \geqslant 2, k \geqslant 2$ and $\ell \geqslant 1$, the split-extension group $H_{n}(k, \ell)$ is the fundamental group of the orbifold $\mathcal{O}((4 k \ell-1) / 2 \ell, n)$ with the 3 -sphere as underlying space and the 2-bridge knot $(4 k \ell-1) / 2 \ell$ with index $n$ as singular set.

First proof. From the presentation of $G_{n}(k, \ell)$ given in Corollary 3.2, we get the following presentation of $H_{n}(k, \ell)$ with $t=t_{1}$ and $q=q_{1}$ :

$$
\begin{aligned}
H_{n}(k, \ell)=\left\langle\rho, t, q: \rho^{n}=1,\left(\rho^{-1} t \rho\right) t^{-1} \rho q^{-1} \rho^{-1}\right. & =1, \\
& \left.\left(q^{k} \rho q^{-(k-1)} \rho^{-1} t \rho^{-1} t^{-1} \rho\right)^{\ell} \rho^{-1} t \rho=1\right\rangle .
\end{aligned}
$$

Hence we have

$$
\begin{aligned}
H_{n}(k, \ell)=\left\langle\rho, t, q: \rho^{n}=1,\left(t^{-1} \rho\right)^{-1} \rho\left(t^{-1} \rho\right)\right. & =\rho q, \\
& \left.\left(q^{k} \rho q^{-(k-1)} \rho^{-1} t \rho^{-1} t^{-1} \rho\right)^{\ell} \rho^{-1} t \rho=1\right\rangle .
\end{aligned}
$$

The element $\mu=\rho q$ is conjugate to $\rho$ from the first relation

$$
\mu=\left(t^{-1} \rho\right)^{-1} \rho\left(t^{-1} \rho\right)
$$

and so $\mu^{n}=1$. Then we have $q=\rho^{-1} \mu$, and

$$
\left(\left(\rho^{-1} \mu\right)^{k} \rho\left(\rho^{-1} \mu\right)^{-(k-1)} \mu^{-1}\right)^{\ell} \mu=t^{-1} \rho .
$$

Thus we obtain a 2 -generator presentation for the group $H_{n}(k, \ell)$ :

$$
H_{n}(k, \ell)=\left\langle\rho, \mu: \rho^{n}=\mu^{n}=1, \rho u=u \mu\right\rangle,
$$




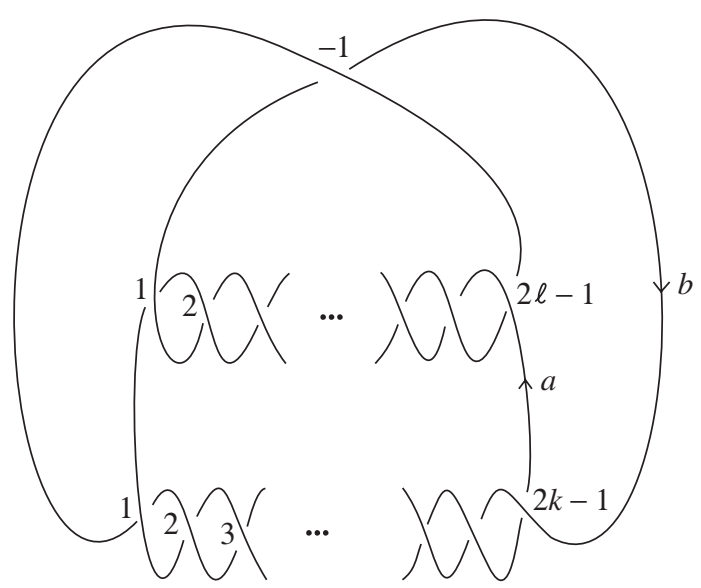

Figure 7 . The 2-bridge knot $(4 k \ell-1) / 2 \ell$.

where

$$
\begin{aligned}
u & =\left[\left(\rho^{-1} \mu\right)^{k} \rho\left(\rho^{-1} \mu\right)^{-(k-1)} \mu^{-1}\right]^{\ell} \\
& =\left[\left(\rho^{-1} \mu\right)^{k} \rho\left(\rho^{-1} \mu\right)^{-k} \rho^{-1}\right]^{\ell} \\
& =\left[\left(\rho^{-1} \mu\right)^{k}, \rho\right]^{\ell} .
\end{aligned}
$$

Since $u$ coincides with the sequence $\rho^{\epsilon_{1}} \mu^{\epsilon_{2}} \cdots \mu^{\epsilon_{4 k \ell-4}} \rho^{\epsilon_{4 k \ell-3}} \mu^{\epsilon_{4 k \ell-2}}$, where the exponent $\epsilon_{i}$ is the sign \pm 1 of $2 i \ell$ reduced $\bmod 8 k \ell-2$ in the interval $(-(4 k \ell-1), 4 k \ell-1)$, the word $u$ corresponds to the 2 -bridge knot $(4 k \ell-1) / 2 \ell$. In particular, the group $\langle\rho, \mu: \rho u=u \mu\rangle$ is isomorphic to the knot group of $(4 k \ell-1) / 2 \ell$, and the generator $\rho$ corresponds to the meridian. Therefore, the group $H_{n}(k, \ell)$ is isomorphic to the fundamental group of the orbifold $\mathcal{O}((4 k \ell-1) / 2 \ell, n)$. We recall that the presentation

$$
\left\langle a, b: b\left[\left(b^{-1} a\right)^{k}, b\right]^{\ell}=\left[\left(b^{-1} a\right)^{k}, b\right]^{\ell} a\right\rangle
$$

defines the group of the 2 -bridge knot $(4 k \ell-1) / 2 \ell$, where $a$ and $b$ correspond to the labels used in Figure 7.

Second proof. From the presentation $P_{n}^{\prime \prime}(k, \ell)$ (see $\S 1$ ), we get

$$
\left(y_{0}^{\ell} y_{1}^{-\ell}\right)^{k}\left(y_{2}^{\ell} y_{1}^{-\ell}\right)^{k} y_{1}=1
$$

hence

$$
\begin{aligned}
H_{n}(k, \ell) & =\left\langle\rho, y: \rho^{n}=1, \quad\left(y^{\ell} \rho^{-1} y^{-\ell} \rho\right)^{k} \rho^{-1}\left(\rho^{-1} y^{\ell} \rho y^{-\ell}\right)^{k} y \rho=1\right\rangle \\
& =\left\langle\rho, y: \rho^{n}=1,\left[y^{\ell}, \rho^{-1}\right]^{k} \rho^{-1}=\rho^{-1} y^{-1}\left[y^{\ell}, \rho^{-1}\right]^{k}\right\rangle .
\end{aligned}
$$

Setting $\lambda=y \rho$, we see that $\lambda$ is conjugate with $\rho$ because $\lambda v=v \rho$, where $v=\left[y^{\ell}, \rho^{-1}\right]^{k}$, hence $\lambda^{n}=1$. So we get

$$
H_{n}(k, \ell)=\left\langle\lambda, \rho: \rho^{n}=\lambda^{n}=1, \lambda v=v \rho\right\rangle,
$$

where $v=\left[\left(\lambda \rho^{-1}\right)^{\ell}, \rho^{-1}\right]^{k}$. Now the proof can be completed as above. 

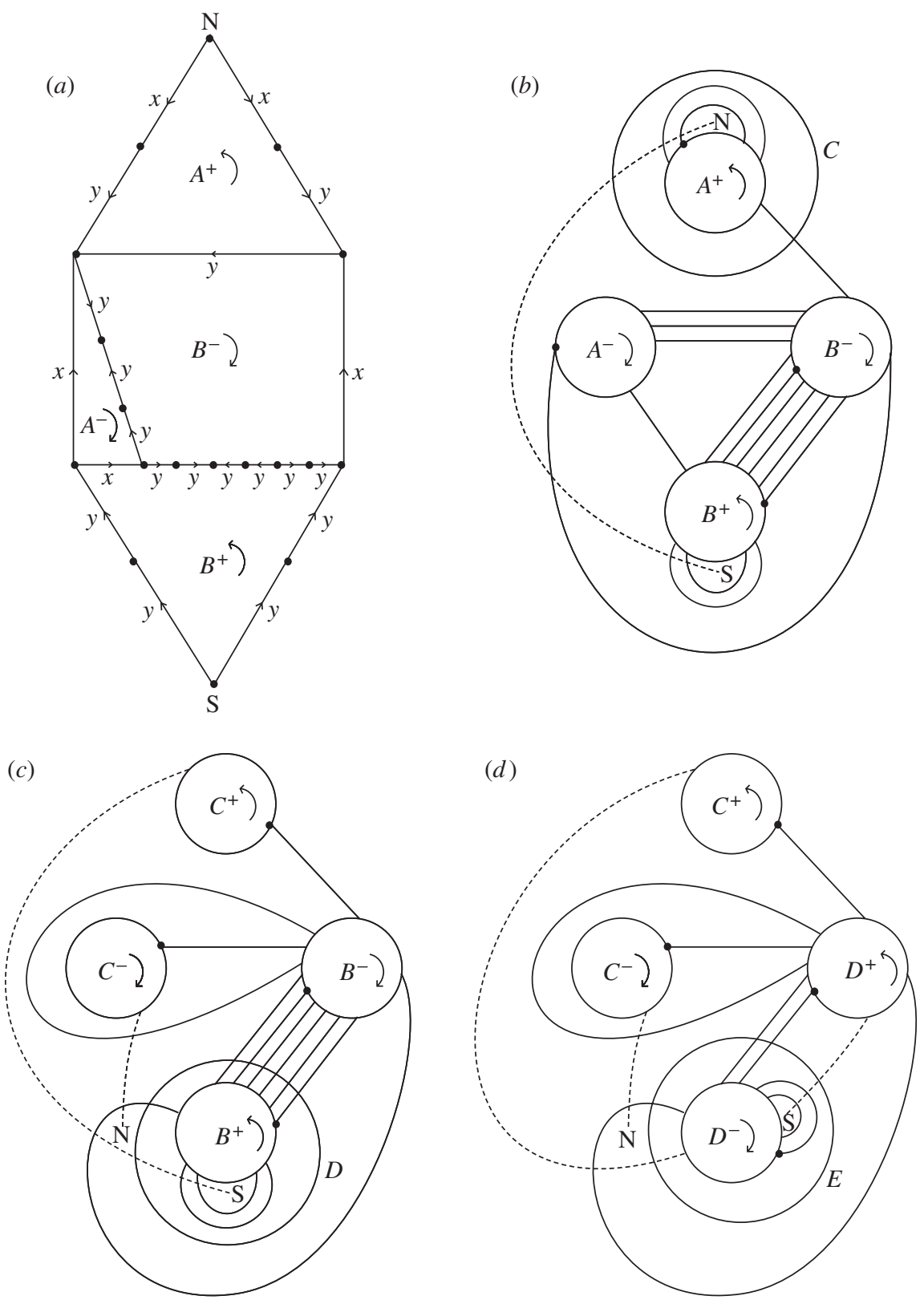

Figure 8. Simplifications along closed curves.

Theorem 4.2. For any $n \geqslant 2, k \geqslant 2$, and coprime integers $p, \ell, 1 \leqslant p<\ell$, the manifold $M_{n}(k, \ell, p)$ is the $n$-fold cyclic covering of the lens space $L(p, \ell)$ branched over a knot which is the image of the south-north axis of the polyhedron $\mathbb{P}_{n}(k, \ell, p)$ under rotation of angle $2 \pi / n$. Furthermore, the manifold $M_{n}(k, \ell)$ is the $n$-fold cyclic covering of the 

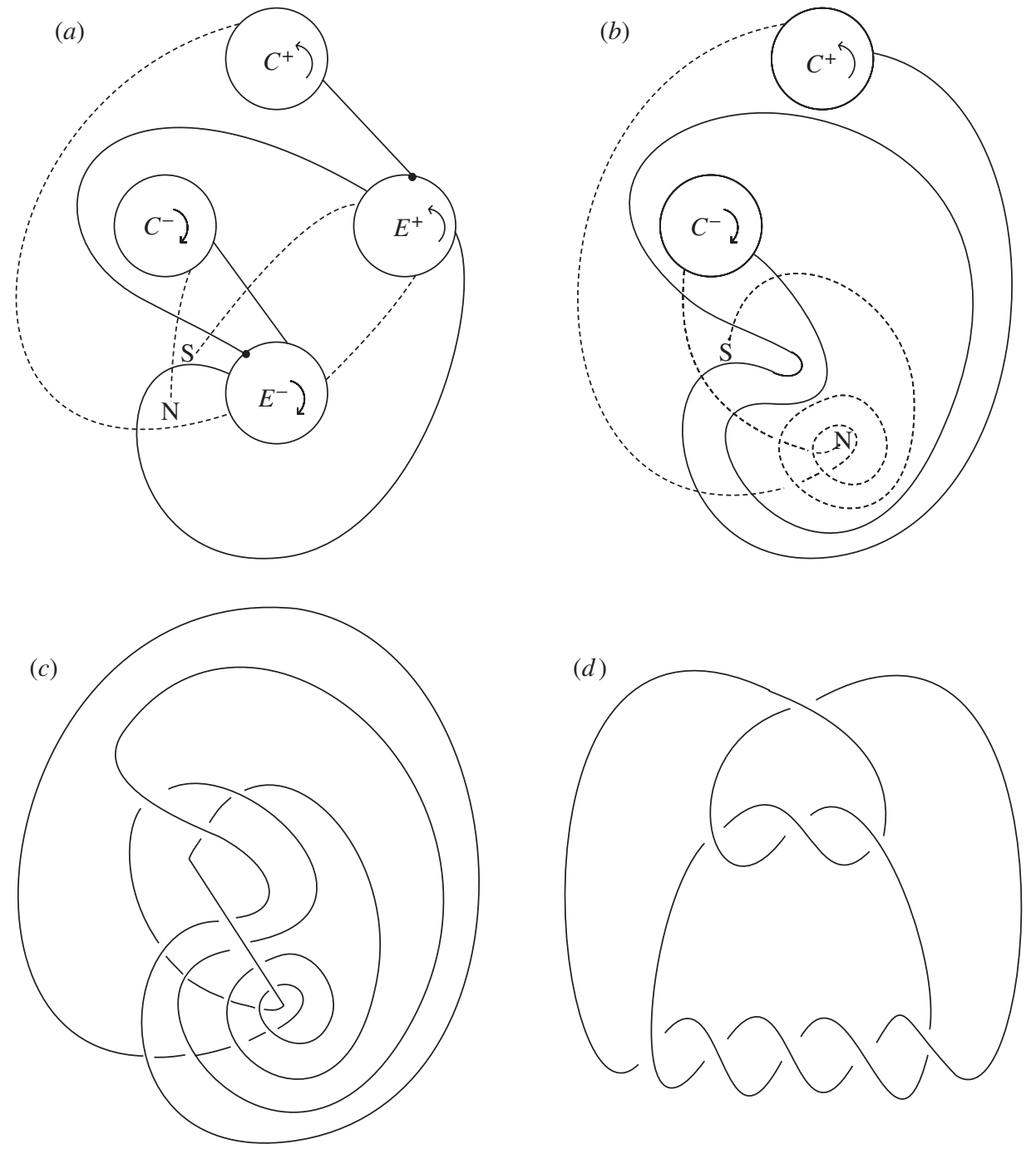

Figure 9. Cancellations of handles.

3 -sphere branched over the 2-bridge knot $(4 k \ell-1) / 2 \ell$, that is, $M_{n}(k, \ell)$ is homeomorphic to the Minkus manifold $M_{n}(4 k \ell-1,2 \ell)$ constructed in [19].

Proof. The manifolds $M_{n}(k, \ell, p)$ are $n$-fold coverings of an orbifold with underlying topological space $M_{1}(k, \ell, p)$ and singular set the image of the NS-axis of the polyhedron $\mathbb{P}_{n}(k, \ell, p)$ under the rotational symmetry of order $n$. To prove the first part of the statement, we consider the polyhedral representation of $M_{1}(k, \ell, p)$, and construct a Heegaard diagram of the manifold directly deduced from the scheme. It is easy to see that this diagram represents the lens space $L(p, \ell)$. Now it remains to analyse the case $p=1$. Rotation by $2 \pi / n$ about the axis NS of the polyhedron $\mathbb{P}_{n}(k, \ell)$ defines an action of $\mathbb{Z}_{n}$ 
on $M_{n}(k, \ell)$. The quotient of the action is the 3 -sphere and the image of the axis NS is a knot $K$. The isotropy group of a point of $M_{n}(k, \ell)$ not on NS is trivial. The quotient $M_{n}(k, \ell) / \mathbb{Z}_{n}$ is obtained by taking a fundamental domain for the action of $\mathbb{Z}_{n}$ on $M_{n}(k, \ell)$ and making identifications (see Figure $8 a$ for $k=3$ and $\ell=2$ ). A Heegaard diagram for this quotient is pictured in Figure $8 b$ and the axis of the rotation is drawn as a dotted curve. Now we apply the method used in [9] for the figure-eight knot (and subsequently extended in $[\mathbf{7}]$ ) to our case. Then we modify Figure $8 b$ to Figure $9 b$ by simplifications along closed curves and cancellations of handles. Figure $8 c$ is obtained from Figure $8 b$ by a simplification along the closed simple curve $C$ (also called a Whitehead-Zieschang reduction). Figure $8 d$ (respectively, Figure $9 a$ ) is obtained from Figure $8 c$ (respectively, Figure $8 d$ ) by a simplification along the closed simple curve $D$ (respectively, $E$ ). Figure $9 b$ (respectively, Figure $9 c$ ) is obtained from Figure $9 a$ (respectively, Figure $9 b$ ) by a cancellation of handles. By using Reidemeister moves, it is easy to see that the knot in Figure $9 c$ is equivalent to the knot $(4 k \ell-1) / 2 \ell$ (which is $23 / 4$ in our example) shown in Figure $9 d$.

Theorem 4.2 says that the manifold $M_{n}(k, \ell)$ is the $n$-fold cyclic covering of the orbifold $\mathcal{O}((4 k \ell-1) / 2 \ell, n)$. By [10], this orbifold is hyperbolic for any $n \geqslant 3$ if $k \geqslant 2$ or $\ell \geqslant 2$. So we immediately obtain the following consequences.

Proposition 4.3. For any $n \geqslant 3$, the manifold $M_{n}(k, \ell), k \geqslant 2, \ell \geqslant 1$, is hyperbolic. Furthermore, $M_{2}(k, \ell)$ is the lens space $L(4 k \ell-1,2 \ell)$, and $M_{1}(k, \ell)$ is the 3 -sphere (hence they have spherical structures).

Proposition 4.4. For any $n \geqslant 3$, the group $G_{n}(k, \ell), k \geqslant 2, \ell \geqslant 1$, is infinite and torsion free, $G_{2}(k, \ell)$ is the cyclic group of order $4 k \ell-1$, and $G_{1}(k, \ell)$ is trivial.

Final remark.

The complete classification of the geometry of the manifolds $M_{n}\left(p_{i}, \ell_{i}, k_{i}\right)$ is still an open problem for arbitrary values of the parameters. If $n \leqslant 2$, then they are homeomorphic to Seifert fibred spaces (including the lens spaces and the 3 -sphere), so the geometry is known (see $\S 2$ ). By Proposition 4.3 several manifolds contained in our class have hyperbolic structures. (For a general reference on the geometry and the toplogy of 3-manifolds see, for example, [31].)

Acknowledgements. This work was performed under the auspices of the GNSAGA of the CNR (National Research Council) of Italy, partly supported by the MURST (Ministero per la Ricerca Scientifica e Tecnologica) of Italy within the project 'Proprietà Geometriche delle Varietà Reali e Complesse', and by a research grant of the University of Modena and Reggio Emilia.

\section{References}

1. P. Bandieri, A. C. Kim And M. Mulazzani, On the cyclic coverings of the knot $5_{2}$, Proc. Edinb. Math. Soc. 42 (1999), 575-587.

2. A. CAVicchioli And F. Spaggiari, The classification of 3-manifolds with spines related to Fibonacci groups, in Algebraic Topology, Homotopy and Group Cohomology, San Feliu de Guixols, 1990, Lecture Notes in Mathematics, vol. 1509, pp. 50-78 (Springer, 1992). 
3. A. Cavicchioli, F. Hegenbarth and A. C. Kim, A geometric study of Sieradski groups, Algebra Colloq. 5 (1998), 203-217.

4. A. Cavicchioli, F. Hegenbarth and A. C. Kim, On cyclic branched coverings of torus knots, J. Geom. 64 (1999), 55-66.

5. A. Cavicchioli, B. Ruini and F. Spaggiari, Cyclic branched coverings of 2-bridge knots, Rev. Mat. Complut. 12(2) (1999), 383-416.

6. A. Cavicchioli, D. Repovš and F. Spaggiari, Topological properties of cyclically presented groups, J. Knot Theory Ramificat. 12(2) (2003), 243-268.

7. F. GRUNEWALD AND U. HiRsCh, Link complements arising from arithmetic group actions, Int. J. Math. 6(3) (1995), 337-370.

8. H. Helling, A. C. Kim and J. L. Mennicke, A geometric study of Fibonacci groups, J. Lie Theory 8(1) (1998), 1-23.

9. H. M. Hilden, M. T. Lozano And J. M. Montesinos-Amilibia, The arithmeticity of the figure eight knot orbifolds, in Topology 90 (ed. B. Apanasov, W. D. Neumann, A. W. Reid and L. Siebenmann), Ohio State University Mathematics Research Institute Publications, vol. 1, pp. 169-183 (Walter de Gruyter, Berlin, 1992).

10. H. M. Hilden, M. T. Lozano And J. M. Montesinos-Amilibia, On the arithmetic 2-bridge knots and link orbifolds and a new knot invariant, J. Knot Theory Ramificat. 4 (1995), 81-114.

11. C. Hog-Angeloni, Detecting 3-manifold presentations, in Computational and Geometric Aspects of Modern Algebra, Edinburgh 1998, London Mathematical Society Lecture Notes, vol. 275, pp. 106-119 (Cambridge University Press, 2000).

12. Y. KIM, About some infinite family of 2-bridge knots and 3-manifolds, Int. J. Math. Math. Sci. 24(2) (2000), 95-108.

13. A. C. KIm AND A. VESnin, Cyclically presented groups and Takahashi manifolds as cyclic coverings, in Analysis of discrete groups, II, Kyoto, 1996, Sur. Kōkyūroku 1022 (1997), $200-212$.

14. A. C. KIm And A. Vesnin, The fractional Fibonacci groups and manifolds, Siberian Math. J. 39 (1998), 655-664.

15. G. KIM, Y. Kim And A. Vesnin, The knot $5_{2}$ and cyclically presented groups, J. Korean Math. Soc. 35 (1998), 961-980.

16. C. MACLACHLAN, Generalisations of Fibonacci numbers, groups and manifolds, in Combinatorial and Geometric Groups Theory, Edinburgh 1993 (ed. A. J. Duncan, N. D. Gilbert and J. Howie), London Mathematical Society Lecture Notes, vol. 204, pp. 233-238 (Cambridge University Press, 1995).

17. C. MacLachlan and A. W. Reid, Generalised Fibonacci manifolds, Transform. Groups 2 (1997), 165-182.

18. A. Mednykh And A. Vesnin, Coxeter groups and branched coverings of lens spaces, $J$. Korean Math. Soc. 38 (2001), 1167-1177.

19. J. Minkus, The branched cyclic coverings of 2-bridge knots and links, Memoirs of the American Mathematical Society, vol. 255 (American Mathematical Society, Providence, RI, 1982).

20. J. M. Montesinos-Amilibia, Surgery on links and double branched covers of $\mathbb{S}^{3}$, in Knots, groups and 3-manifolds (ed. L. P. Neuwirth), pp. 227-259 (Princeton University Press, Princeton, NJ, 1975).

21. M. Mulazzani, On periodic Takahashi manifolds, Tsukuba J. Math. 25 (2001), 229-237.

22. R. Osborne, The simplest closed 3-manifolds, Pac. J. Math. 74 (1978), 481-495.

23. R. Osborne And R. S. Stevens, Group presentations corresponding to spines of 3-manifolds, I, Am. J. Math. 96 (1974), 454-471.

24. R. Osborne And R. S. Stevens, Group presentations corresponding to spines of 3-manifolds, II, Trans. Am. Math. Soc. 234 (1977), 213-243. 
25. R. Osborne And R. S. Stevens, Group presentations corresponding to spines of 3-manifolds, III, Trans. Am. Math. Soc. 234 (1977), 245-251.

26. D. Rolfsen, Knots and links, Mathematics Lecture Notes Series, vol. 7 (Publish or Perish, Berkeley, CA, 1976).

27. B. Ruini And F. Spaggiari, On the structure of Takahashi manifolds, Tsukuba J. Math. 22 (1998), 723-739 (Corrigendum: Tsukuba J. Math. 24 (2000), 433-434).

28. P. Scotт, The geometries of 3-manifolds, Bull. Lond. Math. Soc. 15 (1983), 401-487.

29. R. S. Stevens, Classification of 3-manifolds with certain spines, Trans. Am. Math. Soc. 205 (1975), 151-166.

30. M. TAKAHASHI, On the presentations of the fundamental groups of 3-manifolds, Tsukuba J. Math. 13 (1989), 175-189.

31. W. P. Thurston, Three-dimensional geometry and topology (ed. S. Levy), vol. 1, Princeton Mathematics Series, vol. 35 (Princeton University Press, Princeton, NJ, 1997).

32. B. Zimmermann, Hurwitz groups and finite group actions on hyperbolic 3-manifolds, $J$. Lond. Math. Soc. 52(2) (1995), 199-208. 